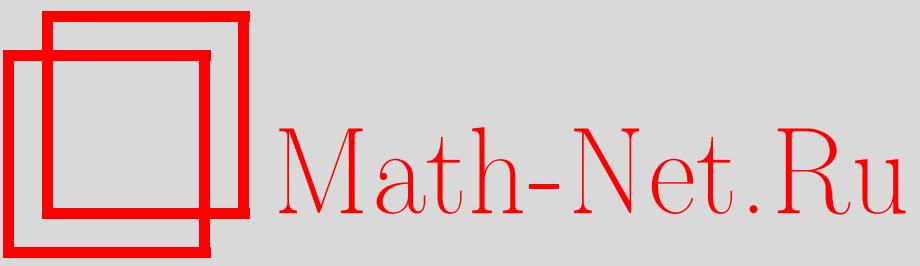

В. Г. Антоник, Сравнение вычислительной эффективности методов градиентного типа в задачах оптимального управления, Итоги науки и техн. Сер. Соврем. мат. и ее прил. Темат. обз., 2020, том 183, 3-13

DOI: https://doi.org/10.36535/0233-6723-2020-183-3-13

Использование Общероссийского математического портала Math-Net.Ru подразумевает, что вы прочитали и согласны с пользовательским соглашением

http://www.mathnet.ru/rus/agreement

Параметры загрузки:

IP : 54.162 .85 .209

26 апреля 2023 г., $14: 35: 28$ 


\title{
СРАВНЕНИЕ ВЫЧИСЛИТЕЛЬНОЙ ЭФФЕКТИВНОСТИ МЕТОДОВ ГРАДИЕНТНОГО ТИПА В ЗАДАЧАХ ОПТИМАЛЬНОГО УПРАВЛЕНИЯ
}

\author{
(c) 2020 г. \\ В. Г. АНТОНИК
}

\begin{abstract}
АннотАция. Оценка эффективности итерационных методов во многом определяется результатами вычислительного эксперимента по решению характерных тестовых и прикладных задач. Статья содержит информацию по численной реализации типовых градиентных методов в сравнении с предложенными авторами модификациями для приближенного решения задач прикладного содержания, известных по литературе (химическая технология, электротехника).
\end{abstract}

Ключевые слова: задача оптимального управления, процедура улучшения, квазиградиентная процедура, вычислительный эксперимент.

\section{COMPARISON OF THE COMPUTATIONAL EFFICIENCY OF GRADIENT-TYPE METHODS IN OPTIMAL CONTROL PROBLEMS}

\author{
(c) 2020 V. G. ANTONIK
}

\begin{abstract}
Estimating of the efficiency of iterative methods is determined by results of computational experiments on solving typical test and applied problems. In this paper, we present information on the numerical implementation of typical gradient methods in comparison with modifications proposed by the authors for the approximate solution of certain applied problems from chemical technology and electrical engineering.
\end{abstract}

Keywords and phrases: optimal control problem, improvement procedure, quasi-gradient procedure, computational experiment.

AMS Subject Classification: 49J15

1. Введение. $\mathrm{K}$ настоящему времени усилиями многих исследователей определились основные классы итерационных методов оптимального управления:

(i) методы принципа максимума (вспомогательная задача на максимум функции Понтрягина, игольчатое варьирование управлений);

(ii) методы градиентного типа (вспомогательная задача дифференциального принципа максимума в линейной или проективной форме, слабое варьирование управлений);

(iii) методы улучшения на основе принципа расширения (линейно-квадратичная аппроксимация функции Кротова, регуляризация целевого функционала как способ варьирования управлений);

(iv) методы дискретизации (конечно-разностная аппроксимация задачи, применение методов математического программирования); 
(v) опорные, корректирующие и адаптивные методы поиска программных и позиционных управлений (кусочно-линейная аппроксимация исходной задачи, асимптотическая коррекция решения линеаризованной задачи);

(vi) методы глобальной оптимизации в невыпуклых задачах специальной структуры (необходимые и достаточные условия оптимальности, разрешающие наборы, улучшение стационарных управлений).

На этом обширном и многогранном итерационном поле градиентные методы (процедуры слабого варьирования) не теряют своей актуальности, сохраняют хорошую репутацию и являются стандартным инструментом численного решения задач оптимального управления. Техника их построения и анализа на вариационном уровне достаточно отработана и вполне соответствует конечномерным аналогам в математическом программировании. Однако в целях повышения эффективности численного решения имеется необходимость более глубокой адаптации градиентной технологии к задачам оптимального управления. Специфика этих задач уже не в первый раз позволяет внести нетривиальные изменения в сложившиеся структуры итерационных процедур и выявить дополнительные резервы повышения качества того или иного метода.

Оценка эффективности итерационных методов во многом определяется результатами вычислительного эксперимента по решению характерных тестовых и прикладных задач. Данная статья содержит информацию по численной реализации предлагаемых методов и модификаций для приближенного решения ряда задач прикладного содержания, известных по литературе (химическая технология, электротехника). Предварительно была проведена алгоритмическая и программная проработка методов (схема численного интегрирования дифференциальных уравнений, выбор констант, стратегия поиска параметров варьирования, условия остановки, компьютерная программа и т. д.). Конечная цель эксперимента состояла, как обычно, в сравнении различных методов (предлагаемых и известных) по некоторым показателям, характеризующим процесс и качество решения задачи (эволюция уменьшения функционала, рекордное значение функционала и соответствующее число задач Коши, итоговые реализации управления и фазовых траекторий). Результирующая картина расчетов не является однозначно определенной, однако доминирующий вывод связан с нетривиальным преимуществом разработанных методов в сравнении с известными процедурами (наилучшее значение функционала, затраты на решение).

2. Постановка задачи. Рассмотрим задачу на минимум целевого функционала

$$
\Phi(u)=\varphi\left(x\left(t_{1}\right)\right)+\int_{T} F(x, u, t) d t \rightarrow \min
$$

относительно фазовой системы

$$
\dot{x}=f(x, u, t), \quad x\left(t_{0}\right)=x^{0},
$$

в классе допустимых управлений

$$
V=\left\{u \in L_{\infty}^{r}(T): u(t) \in U, t \in T\right\} .
$$

Здесь $t$ - независимая переменная, $u(t) \in \mathbb{R}^{r}$ - управление, $x(t) \in \mathbb{R}^{n}$ - фазовое состояние.

Предположим, что в задаче (1)-(3) выполнены следующие условия:

(а) функция $\varphi(x)$ дважды непрерывно дифференцируема на $\mathbb{R}^{n}$, функция $F(x, u, t)$ и векторфункция $f(x, u, t)$ непрерывны по совокупности своих аргументов на $\mathbb{R}^{n} \times U \times T$ вместе с производными по переменной $x$ до второго порядка включительно;

(b) на множестве $\mathbb{R}^{n} \times U \times T$ существуют непрерывные производные $F_{u}(x, u, t), f_{u}(x, u, t)$;

(c) множество $U \subset \mathbb{R}^{r}$ - выпукло и замкнуто.

Будем считать, что каждому допустимому управлению $u \in V$ соответствует единственное абсолютно непрерывное решение $x(t, u), t \in T$, задачи Коши (2). Пару $(u(t), x(t, u)), t \in T$, назовем допустимой в задаче (1)-(3), если $u \in V$, а $x(t, u)$ - соответствующая фазовая траектория. 
Определим необходимые конструкции для задачи (1)-(3). Введем в рассмотрение сопряженную переменную $\psi \in \mathbb{R}^{n}$ и образуем функцию Понтрягина

$$
H(\psi, x, u, t)=\langle\psi, f(x, u, t)\rangle-F(x, u, t) .
$$

Рассмотрим сопряженную задачу

$$
\dot{\psi}=-H_{x}(\psi, x, u, t), \quad \psi\left(t_{1}\right)=-\varphi_{x}\left(x\left(t_{1}\right)\right) .
$$

Сформулируем необходимые условия оптимальности в задаче (1)-(3).

Пусть $(u(t), x(t, u))$ - допустимая пара, $x(t, u)$ - решение сопряженной системы (4) при $u=$ $u(t), x=x(t, u)$. Будем использовать обозначения

$$
H_{u}[t, u]=H_{u}(\psi(t, u), x(t, u), u(t), t), \quad t \in T ;
$$

$\boldsymbol{P}_{U}(\cdot)$ - оператор проектирования на множество $U$ в евклидовой метрике.

Предложение 1 (дифференциальный принцип максимума). Для оптимальности управления $u(t), t \in T$, в задаче (1)-(3) необходимо, чтобъ

$$
u(t)=\boldsymbol{P}_{U}\left(u(t)+\alpha H_{u}[t, u]\right), \quad t \in T, \quad \alpha>0 .
$$

Предложение 2 (условие стационарности). Для оптимальности управления $u(t) \in \operatorname{int} U$, $t \in T$, в задаче (1)-(3) необходимо, чтобь

$$
H_{u}[t, u]=0, \quad t \in T .
$$

Отметим, что вектор-функция $H_{u}[t, u]$ есть антиградиент функционала $\Phi$ на управлении $u(t)$.

Перейдем к описанию процедур улучшения допустимых управлений в поставленной задаче (см. [1-4]).

\section{3. Процедуры улучшения.}

3.1. Прочедуры улучшения в задаче без ограничений. Рассмотрим задачу (1)-(3) без ограничений на управление, когда $U=\mathbb{R}^{r}$. Отметим, что в этом случае условием оптимальности управления $u(t), t \in T$ является соотношение (6).

Приведем общую схему прочедуры слабого улучшения:

(1) по заданному управлению $u \in V$ найдем соответствующие траектории $x(t, u)$ и $\psi(t, u)$;

(2) образуем семейство управлений

$$
u_{\alpha}(t)=u(t)+\alpha s(t), \quad t \in T, \quad \alpha>0 ;
$$

(3) найдем значение параметра $\alpha>0$ из условия улучшения $\Phi\left(u_{\alpha}\right) \leqslant \Phi(u)$.

Укажем конкретные варианты функции $s(t)$ в $(7)$ :

(i) первая прочедура слабого улучшения $\left(\Pi_{0}^{(\infty)}\right)$ :

$$
s(t)=H_{u}[t, u]
$$

(ii) вторая процедура слабого улучшения $\left(\Pi_{0}^{(2)}\right)$ :

$$
s(t)=\frac{g(t)}{\|g(\cdot)\|_{L_{2}}},
$$

где $g(t)=\left\langle H_{u}[t, u], H_{u}[t, u]\right\rangle-$ функция невязки.

Опишем общую схему модифицированной прочедуры:

1. По допустимой паре $(u(t), x(t, u))$ найдем сопряженную траекторию $\psi(t, u)$.

2. Сформируем $\alpha$-параметрическое семейство управлений

$$
u_{\alpha}(t, x)=u(t)+\alpha s(x, t), \quad x \in \mathbb{R}^{n}, \quad t \in T, \quad \alpha>0 .
$$

3. Найдем траекторию $x_{\alpha}(t), t \in T$, как решение задачи Коши для фазовой системы

$$
\dot{x}=f\left(x, u_{\alpha}(t, x), t\right), \quad x\left(t_{0}\right)=x^{0},
$$

вместе с управлением

$$
v_{\alpha}(t)=u_{\alpha}\left(t, x_{\alpha}(t)\right), \quad t \in T \text {. }
$$


4. Параметр $\alpha>0$ будем искать из условия улучшения $\Phi\left(v_{\alpha}\right) \leqslant \Phi(u)$.

Конкретизируем варианты выбора функции $s(x, t)$ в $(8)$ :

(i) модифицированная процедура первого порядка $\left(\Pi_{1}\right)$ :

$$
s(x, t)=H_{u}(\psi(t, u), x, u(t), t) ;
$$

(ii) модифицированная прочедура второго порядка $\left(\Pi_{2}\right)$ :

$$
s(x, t)=H_{u}(p(t, u, x), x, u(t), t),
$$

где

$$
p(t, u, x)=\psi(t, u)+\Psi(t, u)(x-x(t, u)),
$$

а $(n \times n)$ симметричная матричная функция $\Psi(t, u)$ является решением матричной задачи Коши

$$
\dot{\Psi}=-f_{x}[t, u]^{T} \Psi-\Psi f_{x}[t, u]-H_{x x}(\psi, x(t, u), u(t), t), \quad \Psi\left(t_{1}\right)=-\varphi_{x x}\left(x\left(t_{1}, u\right)\right) .
$$

Сформулируем общий результат о возможности улучшения для указанных процедур.

Предложение 3. Если базовое управление и $(t)$ не является стационарным, то $\Phi(w)<\Phi(u)$ для малых $\alpha>0$, где $w=u_{\alpha} \vee v_{\alpha}$.

3.2. Проективные методы улучшения в задаче с ограничениями. Рассмотрим задачу (1)-(3) в исходной постановке ( $U$ - выпуклое замкнутое множество). В рамках данной задачи естественно использовать операцию проектирования на множество $U\left(\boldsymbol{P}_{U}\right.$ - оператор проектирования в евклидовой метрике). При этом базовым условием оптимальности является дифференциальный принцип максимума (ДПМ) в проективной форме (5).

Общая схема прочедур проектирования.

1. Пусть $(u(t), x(t, u)), t \in T$, - допустимая пара в задаче $(1)-(3), \psi(t, u), \Psi(t, u)$ - решения векторной и матричной сопряженных систем,

$$
p(t, u, x)=\psi(t, u)+\Psi(t, u)(x-x(t, u)), \quad x \in \mathbb{R}^{n},
$$

- вспомогательная вектор-функция.

2. Для $\alpha>0$ определим процедуру варьирования

$$
u_{\alpha}(t, x)=\boldsymbol{P}_{U}(u(t)+\alpha s(x, t)) ;
$$

3. Найдем решение $x_{\alpha}(t), t \in T$ фазовой системы

$$
\dot{x}=f\left(x, u_{\alpha}(t, x), t\right), \quad x\left(t_{0}\right)=x^{0},
$$

вместе с управлением $v_{\alpha}(t)=u_{\alpha}\left(t, x_{\alpha}(t)\right), t \in T$;

4. Определим задачу поиска параметра $\alpha$ условием улучшения функционала

$$
\Phi\left(v_{\alpha}\right) \leqslant \Phi(u), \quad \alpha>0 .
$$

Приведем варианты функции $s(x, t)$ в $(9)$ :

(i) метод проектирования первого порядка:

$$
s(x, t)=H_{u}(\psi(t, u), x, u(t), t) ;
$$

(ii) метод проектирования второго порядка:

$$
s(x, t)=H_{u}(p(t, u, x), x, u(t), t) .
$$

Сформулируем основное утверждение о локальном уменьшении целевого функционала.

Предложение 4. Если управление $u \in V$ не удовлетворяет ДПМ в задачеЁ(1)-(3), то проективные методы первого и второго порядка обеспечивают локальное улучшение для малых $\alpha>0$ :

$$
\Phi\left(v_{\alpha}\right)<\Phi(u) .
$$


3.3. Квазиградиентные прочедуры в задаче с выпуклым и компактным ограничением на управление. Рассмотрим задачу (1)-(3) при дополнительном условии ограниченности множества $U$. Иначе говоря, множество $U$ предполагается выпуклым компактом в $\mathbb{R}^{r}$.

Пусть $u(t), t \in T$, - допустимое управление с траекториями $x(t, u), \psi(t, u)$ фазовой и сопряженной систем. Используя антиградиент $H_{u}[t, u]$ функционала $\Phi$ на управлении $u \in V$, сформулируем соответствующее условие оптимальности.

Предложение 5 (дифференциальный принцип максимума). Для оптимальности управления $u \in V$ в задаче (1)-(3) необходимо, чтобы

$$
u(t)=\arg \max _{v \in U}\left\langle H_{u}[t, u], v\right\rangle, \quad t \in T .
$$

Укажем типовую схему метода условного градиента (МУГ).

1. Ппо допустимой паре $(u(t), x(t, u))$ найдем решение $\psi(t, u)$ сопряженной задачи Коши.

2. Сформируем вспомогательное управление

$$
v(t)=\arg \max _{w \in U}\left\langle H_{u}[t, u], w\right\rangle, \quad t \in T .
$$

3. Построим выпуклую комбинацию управлений

$$
u_{\alpha}(t)=u(t)+\alpha(v(t)-u(t)), \quad t \in T .
$$

4. Параметр $\alpha$ найдем из условия $\Phi\left(u_{\alpha}\right) \leqslant \Phi(u), \alpha \in[0,1]$.

Опишем квазиградиентную схему улучшения допустимых управлений в задаче (1)-(3).

1. Пусть $(u(t), x(t, u))$ - допустимая пара, $\psi(t, u), \Psi(t, u)$ - соответствующие решения векторной и матричной сопряженных систем.

2. Образуем вспомогательное (максимизирующее) управление

$$
v(t, x)=\arg \max _{w \in U}\langle s(x, t), w\rangle, \quad x \in \mathbb{R}^{n}, \quad t \in T
$$

3. Сформируем семейство управлений с параметром $\alpha$ :

$$
u_{\alpha}(t, x)=u(t)+\alpha(v(t, x)-u(t)), \quad t \in T .
$$

4. Решая задачу Коши

$$
\dot{x}=f\left(x, u_{\alpha}(t, x), t\right), \quad x\left(t_{0}\right)=x^{0},
$$

найдем фазовую траекторию $x_{\alpha}(t), t \in T$, вместе с управлением $v_{\alpha}(t)=u_{\alpha}\left(t, x_{\alpha}(t)\right), t \in T$.

5. Задача поиска параметра $\alpha \in[0,1]$ определяется требованием улучшения целевого функционала: $\Phi\left(v_{\alpha}\right) \leqslant \Phi(u)$.

Как и прежде, конкретный вид процедур улучшения связан с выбором функции $s(x, t)$ в $(10)$ :

(i) первый метод условного квазиградиента (МУК-1):

$$
s(x, t)=H_{u}(\psi(t, u), x, u(t), t) ;
$$

(ii) второй метод условного квазиградиента (МУК-2):

$$
s(x, t)=H_{u}(p(t, u, x), x, u(t), t) .
$$

4. Вычислительный эксперимент. Опишем численные расчеты по реализации предлагаемых методов и модификаций в сравнении с известными вариантами и между собой. Были использованы конкретные задачи с различными особенностями, известные по литературе в плане их численного решения с помощью тех или иных методов. В разделе 4.1 рассмотрены задачи без ограничений на управление, для решения которых были апробированы представленные выше процедуры градиентного и квазиградиентного спуска. Раздел 4.2 содержит задачи с ограничениями, в рамках которых проведена сравнительная реализация методов условного квазиградиента вместе с процедурой проектирования.

Полученные результаты сравнительной эффективности достаточно корректны, поскольку расчеты проводились фактически по одной программе, т.е. в идентичных условиях. В качестве единицы трудоемкости методов традиционно использована задача Коши для системы $n$ уравнений, 
т.е. критерием эффективности при прочих равных показателях является число задач Коши, необходимое для приближенного решения задачи данным методом. При этом в качестве условия остановки методов для каждой задачи использовалась оценка по невязке соответствующего условия оптимальности с единой константой в правой части.

\section{1. Задачи без ограничений на управление.}

Задача 1 (см. [6, 7]). Рассмотрим билинейно-квадратичную задачу без ограничений на управление, которая моделирует экзотермический процесс в химическом реакторе с непрерывным перемешиванием продукта:

$$
\begin{array}{cl}
\Phi(u)=x_{1}^{2}(3)+x_{2}^{2}(3)+\frac{1}{2} \int_{0}^{3}\left[10\left(x_{1}^{2}(t)+x_{2}^{2}(t)\right)+u^{2}(t)\right] d t \rightarrow \text { min }, \\
\left\{\begin{array}{lcc}
\dot{x}_{1}=\frac{13}{6} x_{1}+\frac{5}{12} x_{2}-x_{1} u-\frac{1}{8} u, & x_{1}(0)=0,15, & t \in[0,3] . \\
\dot{x}_{2}=-\frac{50}{3} x_{1}-\frac{8}{3} x_{2}, & x_{2}(0)=0,
\end{array}\right.
\end{array}
$$

Здесь безразмерные фазовые переменные $x_{1}, x_{2}$ характеризуют температуру и концентрацию начального продукта реакции, безразмерное управление $u$ описывает интенсивность процесса внешнего охлаждения. Функционал качества характеризует среднее квадратичное отклонение текущего состояния $\left(x_{1}(t), x_{2}(t), u(t)\right)$ от желаемого стационарного (магистрального) режима $x(t)=0, u(t)=0$.

В данной задаче функция Понтрягина имеет вид

$$
H=\psi_{1}\left(\frac{13}{6} x_{1}+\frac{5}{12} x_{2}-x_{1} u-\frac{1}{8} u\right)+\psi_{2}\left(-\frac{50}{3} x_{1}-\frac{8}{3} x_{2}\right)-\frac{1}{2}\left(10\left(x_{1}^{2}+x_{2}^{2}\right)+u^{2}\right),
$$

первая сопряженная система

$$
\begin{cases}\dot{\psi}_{1}=-\frac{13}{6} \psi_{1}+u \psi_{1}+\frac{50}{3} \psi_{2}+10 x_{1}, & \psi_{1}(3)=-2 x_{1}(3) \\ \dot{\psi}_{2}=-\frac{5}{12} \psi_{1}+\frac{8}{3} \psi_{2}+10 x_{2}, & \psi_{2}(3)=-2 x_{2}(3)\end{cases}
$$

вторая сопряженная система

$$
\begin{cases}\dot{\Psi}_{11}=\left(2 u-\frac{13}{3}\right) \Psi_{11}+\frac{100}{3} \Psi_{12}+10, & \Psi_{11}(3)=-2 \\ \dot{\Psi}_{12}=-\frac{5}{12} \Psi_{11}+\left(\frac{1}{2}+u\right) \Psi_{12}+\frac{50}{3} \Psi_{22}, & \Psi_{12}(3)=0 \\ \dot{\Psi}_{22}=-\frac{5}{6} \Psi_{12}+\frac{16}{3} \Psi_{22}+10, & \Psi_{22}(3)=-2\end{cases}
$$

градиент функционала определяется производной

$$
-H_{u}=\psi_{1}\left(x_{1}+\frac{1}{8}\right)+u
$$

Решение задачи проведено с помощью процедур слабого улучшения $\Pi_{0}^{(\infty)}$ и $\Pi_{0}^{2}$, а также модифицированной процедуры второго порядка $\Pi_{2}$.

Поиск параметра варьирования $\alpha>0$ производился по способу половинного деления $\left(\alpha_{0}=1\right.$, $\left.\alpha_{k+1}=\alpha_{k} / 2, k=0,1, \ldots\right)$. Условие окончания процедуры $\alpha$-поиска:

$$
\Phi\left(u_{\alpha_{k}}\right)<\min \left\{\Phi\left(u_{\alpha_{k-1}}\right), \Phi(u)\right\}, \quad \Phi\left(u_{\alpha_{k+1}}\right)>\Phi\left(u_{\alpha_{k}}\right), \quad k=1,2, \ldots
$$

( $\alpha_{k}$-итоговое значение параметра, половинное деление производится до тех пор, пока функционал уменьшается). 
Условие остановки метода имеет вид

$$
\delta_{0}(u)=\int_{0}^{3} g(t) d t \leqslant \varepsilon, \quad \varepsilon=10^{-2} .
$$

Шаг интегрирования дифференциальных систем равен $h=0,006$, начальное управление $u^{0}(t)=0$.

Результаты расчетов отражены в следующей таблице, где $\Phi_{*}$ - наилучшее расчетное значение функционала, $\mathcal{N}$ - общее число задач Коши для фазового и сопряженных уравнений, $\mathcal{M}-$ количество итераций:

\begin{tabular}{|c|c|c|c|}
\hline & $\Phi_{*}$ & $\mathcal{N}$ & $\mathcal{M}$ \\
\hline$\Pi_{0}^{(\infty)}$ & 0,93091 & 219 & 27 \\
\hline$\Pi_{0}^{(2)}$ & 0,93049 & 111 & 14 \\
\hline$\Pi_{2}$ & 0,93031 & 153 & 20 \\
\hline
\end{tabular}

Bъводъ. По вычислительным затратам наилучший результат убедительно показала процедура $\Pi_{0}^{(2)}$. Модификация $\Pi_{2}$ обеспечивает наилучшее значение функционала. Стандартный метод $\Pi_{0}^{(\infty)}$ в данном случае проигрывает по всем показателям.

Задача 2 (см. [7]). Рассмотрим следующую билинейно-квадратичную задачу с двумя управлениями:

$$
\begin{aligned}
\Phi(u)= & \frac{1}{2}\left(x_{1}^{2}(10)+x_{2}^{2}(10)\right)+\frac{1}{2} \int_{0}^{10}\left(u_{1}^{2}(t)+u_{2}^{2}(t)\right) d t \rightarrow \min , \\
& \begin{cases}\dot{x}_{1}=u_{1} x_{2}, & x_{1}(0)=0,1, \\
\dot{x}_{2}=u_{2}, & x_{2}(0)=1,\end{cases}
\end{aligned}
$$

В данной задаче функция Понтрягина имеет вид

$$
H=\psi_{1} u_{1} x_{2}+\psi_{2} u_{2}-\frac{1}{2}\left(u_{1}^{2}+u_{2}^{2}\right),
$$

первая сопряженная система

$$
\begin{cases}\dot{\psi}_{1}=0, & \psi_{1}(10)=-x_{1}(10), \\ \dot{\psi}_{2}=-\psi_{1} u_{1}, & \psi_{2}(10)=-x_{2}(10),\end{cases}
$$

вторая сопряженная система

$$
\begin{cases}\dot{\Psi}_{11}=0, & \Psi_{11}(10)=-1 \\ \dot{\Psi}_{12}=-u_{1} \Psi_{11}, & \Psi_{12}(10)=0 \\ \dot{\Psi}_{22}=-2 u_{1} \Psi_{12}, & \Psi_{22}(10)=-1 ;\end{cases}
$$

градиент функционала определяется производными

$$
H_{u_{1}}=\psi_{1} x_{2}-u_{1}, \quad H_{u_{2}}=\psi_{2}-u_{2} .
$$

Решение задачи проводилось с помощью процедур $\Pi_{0}^{(\infty)}, \Pi_{0}^{(2)}, \Pi_{2}$. Кроме того была использована модификация первого порядка

$$
\Pi_{1}: u_{\alpha}(t, x)=u(t)+\alpha H_{u}(\psi(t, u), x, u(t), t) .
$$

Подбор параметра $\alpha>0$ проводился аналогично предыдущей задаче. Шаг интегрирования $h=0,02$, параметр остановки $\varepsilon=10^{-2}$, начальное управление $u_{1}^{0}(t)=0, u_{2}^{0}(t)=1$. Результаты расчетов отражены в следующей таблице: 


\begin{tabular}{|c|c|c|c|}
\hline & $\Phi_{*}$ & $\mathcal{N}$ & $\mathcal{M}$ \\
\hline$\Pi_{0}^{(\infty)}$ & 0,04986 & 33 & 5 \\
\hline$\Pi_{0}^{(2)}$ & 0,04766 & 19 & 4 \\
\hline$\Pi_{1}$ & 0,04692 & 41 & 6 \\
\hline$\Pi_{2}$ & 0,048158 & 40 & 7 \\
\hline
\end{tabular}

Bъводы. Эффект модификаций $\Pi_{0}^{(2)}, \Pi_{1}, \Pi_{2}$ в сравнении со стандартным градиентным методом $\Pi_{0}^{(\infty)}$ вполне очевиден как по итоговым значениям функционала, так и по вычислительным затратам. Наилучший результат по затратам дает модификация $\Pi_{0}^{(2)}$. Процедура $\Pi_{1}$ реализует наилучшее значение функционала. Следует отметить «скромный» результат по модификации $\Pi_{2}$.

Задача 3 (см. [8]). Рассмотрим следующую нелинейную задачу без ограничений на управление:

$$
\begin{gathered}
\Phi(u)=\int_{0}^{5}\left(x_{1}^{2}(t)+x_{2}^{2}(t)+u^{2}(t)\right) d t \rightarrow \min , \\
\left\{\begin{array}{ll}
\dot{x}_{1}=x_{2}, & x_{1}(0)=1,5, \\
\dot{x}_{2}=-x_{1}+\left(1-x_{1}^{2}\right) x_{2}+u, & x_{2}(0)=1.5,
\end{array} \quad t \in[0,5] .\right.
\end{gathered}
$$

В данной задаче функция Понтрягина имеет вид

$$
H=\psi_{1} x_{2}+\psi_{2}\left(-x_{1}+\left(1-x_{1}^{2}\right) x_{2}+u\right)-\left(x_{1}^{2}+x_{2}^{2}+u^{2}\right)
$$

первая сопряженная система

$$
\begin{cases}\dot{\psi}_{1}=\psi_{2}+2 x_{1} \psi_{2} x_{2}+2 x_{1}, & \psi_{1}(5)=0 \\ \dot{\psi}_{2}=-\psi_{1}-\psi_{2}+\psi_{2} x_{1}^{2}+2 x_{2}, & \psi_{2}(5)=0\end{cases}
$$

вторая сопряженная система

$$
\left\{\begin{array}{rlrl}
\dot{\Psi}_{11} & =2+4\left(1-x_{1}\right) x_{2} \Psi_{12}-2 \psi_{2} x_{2}+2, & & \Psi_{11}(5)=0 \\
\dot{\Psi}_{12} & =\left(1+2\left(1-x_{1}\right) x_{2}\right) \Psi_{11}-\left(1-x_{1}\right)^{2} \Psi_{12}+ & & \\
& +\left(1+2\left(1-x_{1}\right) x_{2}\right) \Psi_{22}+2 \psi_{2}\left(1-x_{1}\right), & & \Psi_{12}(5)=0 \\
\dot{\Psi}_{22}=-2 \Psi_{12}-2\left(1-x_{1}\right)^{2} \Psi_{22}+2, & & \Psi_{22}(5)=0
\end{array}\right.
$$

градиент функционала определяется производной

$$
H_{u}=\psi_{2}-2 u \text {. }
$$

Решение проводилось с помощью процедур $\Pi_{0}, \Pi_{1}, \Pi_{2}$. Шаг интегрирования $h=0,01$, параметр остановки $\varepsilon=10^{-2}$, начальное управление $u^{0}(t)=1$. Результаты расчетов приведены в следующей таблице:

\begin{tabular}{|c|c|c|c|}
\hline & $\Phi_{*}$ & $\mathcal{N}$ & $\mathcal{M}$ \\
\hline$\Pi_{0}^{(\infty)}$ & 8,78665 & 278 & 35 \\
\hline$\Pi_{0}^{(2)}$ & 8,78651 & 246 & 34 \\
\hline$\Pi_{1}$ & 8,78665 & 278 & 35 \\
\hline$\Pi_{2}$ & 8,78499 & 198 & 25 \\
\hline
\end{tabular}

$B$ ъводы. В данной задаче абсолютный приоритет имеет процедура $\Pi_{2}$. Модификация П почтительнее стандартного варианта $\Pi_{0}^{(\infty)}$. 


\section{2. Задачи с ограничениями на управление.}

Задача 4 (см. [5]). Рассмотрим следующую линейную по состоянию задачу с ограничениями на управление, которая связана с оптимизацией некоторого химического аппарата:

$$
\begin{gathered}
\Phi(u)=-x_{3}(1) \rightarrow \min , \\
\begin{cases}\dot{x}_{1}=-\left(k_{1}(u)+k_{2}(u)+k_{3}(u)\right) x_{1}, & x_{1}(0)=1, \\
\dot{x}_{2}=k_{1}(u) x_{1}-k_{4}(u) x_{2}, & x_{2}(0)=0, \\
\dot{x}_{3}=k_{4}(u) x_{2}-k_{5}(u) x_{3}, & x_{3}(0)=0, \\
u(t) \in[0,823], \quad t \in[0,1] . & \end{cases}
\end{gathered}
$$

Система дифференциальных уравнений описывает реакции, протекающие в смеси трех веществ, $x_{i}(t), i=1,2,3,-$ их концентрации. Интенсивности реакций зависят от температуры $u(t)$, играющей в данной задаче роль управления. Первое вещество, концентрация которого $x_{1}(t),-$ сырье, второе - промежуточный продукт, третье - окончательный продукт.

Функции $k_{i}(u)$ имеют характерный для химической кинетики вид

$$
k_{i}(u)=C_{i} \exp \left[\frac{E_{i}}{R}\left(\frac{1}{658}-\frac{1}{u}\right)\right], \quad i=1, \ldots, 5 .
$$

Значения постоянных таковы:

$$
\begin{gathered}
C_{1}=1,02, \quad C_{2}=0,93, \quad C_{3}=0,386, \quad C_{4}=3,28, \quad C_{5}=0,084, \quad R=1,9865, \\
E_{1}=16000, \quad E_{2}=14000, \quad E_{3}=15000, \quad E_{4}=10000, \quad E_{5}=15000 .
\end{gathered}
$$

В данной задаче функция Понтрягина имеет вид

$$
H=-\psi_{1}\left(k_{1}(u)+k_{2}(u)+k_{3}(u)\right) x_{1}+\psi_{2}\left(k_{1}(u) x_{1}-k_{4}(u) x_{2}\right)+\psi_{3}\left(k_{4}(u) x_{2}-k_{5}(u) x_{3}\right) ;
$$

сопряженная система

$$
\begin{cases}\dot{\psi}_{1}=\psi_{1}\left(k_{1}(u)+k_{2}(u)+k_{3}(u)\right)-\psi_{2} k_{1}(u), & \psi_{1}(1)=0, \\ \dot{\psi}_{2}=\psi_{2} k_{4}(u)-\psi_{3} k_{4}(u), & \psi_{2}(1)=0 \\ \dot{\psi}_{3}=\psi_{3} k_{5}(u), & \psi_{3}(1)=1\end{cases}
$$

градиент функционала определяется производной

$$
H_{u}=-\psi_{1} x_{1}\left(\sum_{i=1}^{3} d_{i}(u)\right)+\psi_{2} x_{1} d_{1}(u)-\psi_{2} x_{2} d_{4}(u)+\psi_{3} x_{2} d_{4}(u)-\psi_{3} x_{3} d_{5}(u)
$$

где

$$
d_{i}(u)=\frac{d k_{i}(u)}{d u}=\frac{E_{i}}{R u^{2}} k_{i}(u), \quad i=1, \ldots, 5 .
$$

Максимизирующее управление ДПМ имеет вид

$$
u^{*}(\psi, x, t)=\arg \max _{v \in[0,823]} H_{u}(\psi, x, u(t)) v= \begin{cases}0, & H_{u}(\psi, x, u(t))<0, \\ 823, & H_{u}(\psi, x, u(t)) \geqslant 0 .\end{cases}
$$

Следует отметить, что в данной задаче методы принципа максимума фактически не реализуемы: условие максимума функции $H$ по управлению аналитически не разрешается.

Задача была решена двумя методами градиентного типа: стандартным методом условного градиента (МУГ) и метод условного квазиградиента первого порядка (MУK-1). Процедура $\alpha$ поиска - метод половинного деления. Начальное управление $u^{0}(t)=600$, шаг интегрирования $h=0,005$, параметр остановки $\varepsilon=10^{-5}$ по невязке ДПМ. Результаты расчетов отражает следующая таблица:

\begin{tabular}{|c|c|c|}
\hline & $\Phi_{*}$ & $\mathcal{N}$ \\
\hline МУГ & $-0,43620$ & 876 \\
\hline МУK-1 & $-0,43682$ & 215 \\
\hline
\end{tabular}


Bъводы. Безусловное преимущество модификации МУК-1 вполне очевидно как по затратам (215 задач Коши против 876), так и по значению функционала.

Задача 5 (см. [8]). Рассмотрим задачу оптимального управления шаговым электродвигателем:

$$
\begin{gathered}
\Phi(u)=\int_{0}^{0,05}\left(x_{1}^{2}+k_{1} u_{1}+k_{2} u_{2}+k_{3} u_{3}\right) d t \rightarrow \min , \\
\begin{cases}\dot{x}_{1}=x_{2}, & x_{1}(0)=\frac{\pi}{3}, \\
\dot{x}_{2}=-a x_{2}-b\left[u_{1} \sin \left(2 x_{1}\right)+u_{2} \sin \left(2 x_{1}+\frac{2 \pi}{3}\right)+u_{3} \sin \left(2 x_{1}-\frac{2 \pi}{3}\right)\right], & x_{2}(0)=0, \\
u_{i}(t) \in[0,16], \quad i=1,2,3, \quad t \in[0,0,05] . & \end{cases}
\end{gathered}
$$

Значения параметров: $k_{i}=0,0014, i=1,2,3, a=50, b=1000$. Здесь $x_{1}$ - положение вала двигателя, $x_{2}$ - его скорость, управления $u_{1}, u_{2}, u_{3}$ соответствуют квадратам токов в обмотках. Функционал $\Phi(u)$ отражает требование стабилизации электродвигателя (приведение положения вала к нулю при минимальных энергозатратах). В данной задаче функция Понтрягина имеет вид

$$
\begin{aligned}
H=\psi_{1} x_{2}-\psi_{2}\left(a x_{2}+b\left[u_{1} \sin \left(2 x_{1}\right)+u_{2} \sin \left(2 x_{1}+\frac{2 \pi}{3}\right)+u_{3} \sin \right.\right. & \left.\left.\left(2 x_{1}-\frac{2 \pi}{3}\right)\right]\right)- \\
& -\left(x_{1}^{2}+k_{1} u_{1}+k_{2} u_{2}+k_{3} u_{3}\right)
\end{aligned}
$$

первая сопряженная система

$$
\begin{cases}\dot{\psi}_{1}=2 b \psi_{2}\left[u_{1} \cos \left(2 x_{1}\right)+u_{2} \cos \left(2 x_{1}+\frac{2 \pi}{3}\right)+u_{3} \cos \left(2 x_{1}-\frac{2 \pi}{3}\right)\right]+2 x_{1}, & \psi_{1}\left(t_{1}\right)=0 \\ \dot{\psi}_{2}=-\psi_{1}+a \psi_{2} & \psi_{2}\left(t_{1}\right)=0\end{cases}
$$

вторая сопряженная система

$$
\left\{\begin{array}{l}
\dot{\Psi}_{11}=4 b \Psi_{12}\left[u_{1} \cos \left(2 x_{1}\right)+u_{2} \cos \left(2 x_{1}+\frac{2 \pi}{3}\right)+u_{3} \cos \left(2 x_{1}-\frac{2 \pi}{3}\right)\right]- \\
\quad-4 b \psi_{2}\left[u_{1} \sin \left(2 x_{1}\right)+u_{2} \sin \left(2 x_{1}+\frac{2 \pi}{3}\right)+u_{3} \sin \left(2 x_{1}-\frac{2 \pi}{3}\right)\right]+2 \\
\dot{\Psi}_{12}=2 b \Psi_{22}\left[u_{1} \cos \left(2 x_{1}\right)+u_{2} \cos \left(2 x_{1}+\frac{2 \pi}{3}\right)+u_{3} \cos \left(2 x_{1}-\frac{2 \pi}{3}\right)\right]-\Psi_{11}+\Psi_{12} a \\
\dot{\Psi}_{22}=-2 \Psi_{12}+2 a \Psi_{22} \\
\quad \Psi_{i j}\left(t_{1}\right)=0, \quad i, j=1,2
\end{array}\right.
$$

градиент функционала определяется производными

$$
H_{u_{1}}=-\psi_{2} b \sin \left(2 x_{1}\right)-k_{1}, \quad H_{u_{2}}=-\psi_{2} b \sin \left(2 x_{1}+\frac{2 \pi}{3}\right)-k_{2}, \quad H_{u_{3}}=-\psi_{2} b \sin \left(2 x_{1}-\frac{2 \pi}{3}\right)-k_{3} .
$$

Максимизирующее управление имеет вид

$$
u_{i}^{*}\left(\psi_{2}, x_{1}\right)=\left\{\begin{array}{ll}
0, & H_{u_{i}}\left(\psi_{2}, x_{1}\right)<0, \\
16, & H_{u_{i}}\left(\psi_{2}, x_{1}\right) \geqslant 0
\end{array} \quad i=1,2,3 .\right.
$$


Решение задачи было проведено с помощью методов МУГ, МУК-1. Кроме того, был использован метод условного квазиградиента второго порядка (МУК-2):

$$
\begin{gathered}
u_{i}(t, x, \alpha)=u_{i}(t)+\alpha\left(v_{i}^{*}(t, x)-u_{i}(t)\right), \quad i=1,2,3, \\
v_{i}^{*}(t, x(t))=u_{i}^{*}\left(p_{2}(t, u, x), x_{1}\right), \\
p_{2}(t, u, x)=\psi_{2}(t, u)+\sum_{j=1}^{2} \Psi_{2 j}(t, u)\left(x_{j}-x_{j}(t, u)\right) .
\end{gathered}
$$

Начальное управление $u^{0}(t)=1$, шаг интегрирования $h=0,00025$, параметр $\alpha$ ищется методом половинного деления из условия монотонного убывания функционала, параметр остановки $\varepsilon=10^{-7}$. Результаты расчетов отражает следующая таблица:

\begin{tabular}{|c|c|c|}
\hline & $\Phi_{*}$ & $\mathcal{N}$ \\
\hline МУГ & 0,00817 & 617 \\
\hline МУK-1 & 0,00988 & 410 \\
\hline МУК-2 & 0,00792 & 287 \\
\hline
\end{tabular}

Bъъодъ. Первая модификация (МУК-1) предпочтительнее стандартного варианта по затратам, однако проигрывает по значению функционала. Безусловный приоритет имеет в данном случае MУK-2.

\section{СПИСОК ЛИТЕРАТУРЫ}

1. Антоник В. Г., Срочко В. А. Метод проекций в линейно-квадратичных задачах оптимального управления// Ж. вычисл. мат. мат. физ. - 1998. - 38, № 4. - С. 564-572.

2. Мамонова H. В., Срочко В. А. Метод проекций в линейно-квадратичных задачах оптимального управления// Изв. вузов. Мат. - 2001. - № 12. - С. 55-67.

3. Срочко B. А. Итерационные методы решения задач оптимального управления. - М.: Физматлит, 2000.

4. Срочко В. А. Модернизация методов градиентного типа в задачах оптимального управления// Изв. вузов. Мат. - 2002. - № 12 . - С. $66-78$.

5. Федоренко Р. П. Приближенное решение задач оптимального управления. - М.: Наука, 1978.

6. Aganovic Z., Gajic Z. The successive approximation procedure for finite-time optimal control of bilinear systems// IEEE Trans. Automat. Control. — 1994. — 39, № 9. — P. 1932-1935.

7. Hofer E. P., Tibken B. An iterative method for the finite-time bilinear-quadratic control problem// J. Optim. Theory Appl. - 1988. - 57, № 3. - P. 411-426.

8. Jones D. I., Finch J. W. Comparison of optimization algorithms// Int. J. Control. — 1984. — 40, № 4. P. $747-761$.

Антоник Владимир Георгиевич

Иркутский государственный университет

E-mail: vga@math.isu.ru 